\title{
Designing and Implementing of Online Message System Based on XML Technology \\ Jing Dong
}

School of Information Engineering, Qujing Normal University, Qujing, China

38841642@qq.com

Keywords: XML; DOM; UBB; Online message

\begin{abstract}
Using XML file as data source and using DOM for the object of the XML data interface, interactive page is achieved by using ASP. In order to achieve online message editing, UBB code is embedded of the system, so online message system based on XML Technology is achieved. The solution is proved to be feasible, high efficiency resource sharing and high reliability.
\end{abstract}

\section{Introduction}

Since XML (Markup Language eXtensible) is widely used launched by W3C, because of its outstanding performance in the Web information exchange and information sharing, especially the exchange of data description and data of all kinds of documents and information in the field of digital library[1], has become an important means of data transmission on the internet.

In nowadays popular relational database, relational data is the information that the structure is fixed. Relation between tables is used to express the relationship between the data, and the XML data is semi-structured information compared with the traditional relational database, the relationship between the elements shows through the nested expression, and the expression form is very flexible[2,3]. Database manage system isn't needed to store data, in addition, in any application can read and write data, it is very simple and convenient. So currently it's powerful tool to process structured document information, what's more, the unified data format is also the future of the web information organization and information exchange trend [4].

XML document has extensible attribute that allows users to customize these tags and labels of related parameters and values; it has good data reusability; also the XML document has a strong independent platform. XML can run on any platform and operating system, has a structured and standardized file mode; finally, the data can be identified uniquely by XML, search software without knowing the construction is similar to the traditional database, so the search will become very easy. The data do not need to use the database system, any application can read and write data, it is very simple and convenient, and has the unified data format, due to its efficient operation and maintenance of Web with the exponential growth of data; it has become an important means of data transmission on network.

\section{System Architecture Design}

Design Ideas. The system uses XML technology to store and manage data, for XML can link the data together in different formats easily[5,6], and Xquery language based on XML has improved, using XML technology to implement online message system, because of its high integration, less code, fast development speed, low maintenance cost, software code takes fewer resources and more operable[7]. The architecture of this system is belonged to Browser/Server architecture, the client does not need to download and install the plug-in, the browser as the client is only responsible for sending the user's request to the IIS server and the server application handle related services requests by client. Interactive page part used ASP to implement for advantage of page elements and code separation, the structure is clear. The system combines the XML with the server side script. System architecture principle as shown below: 


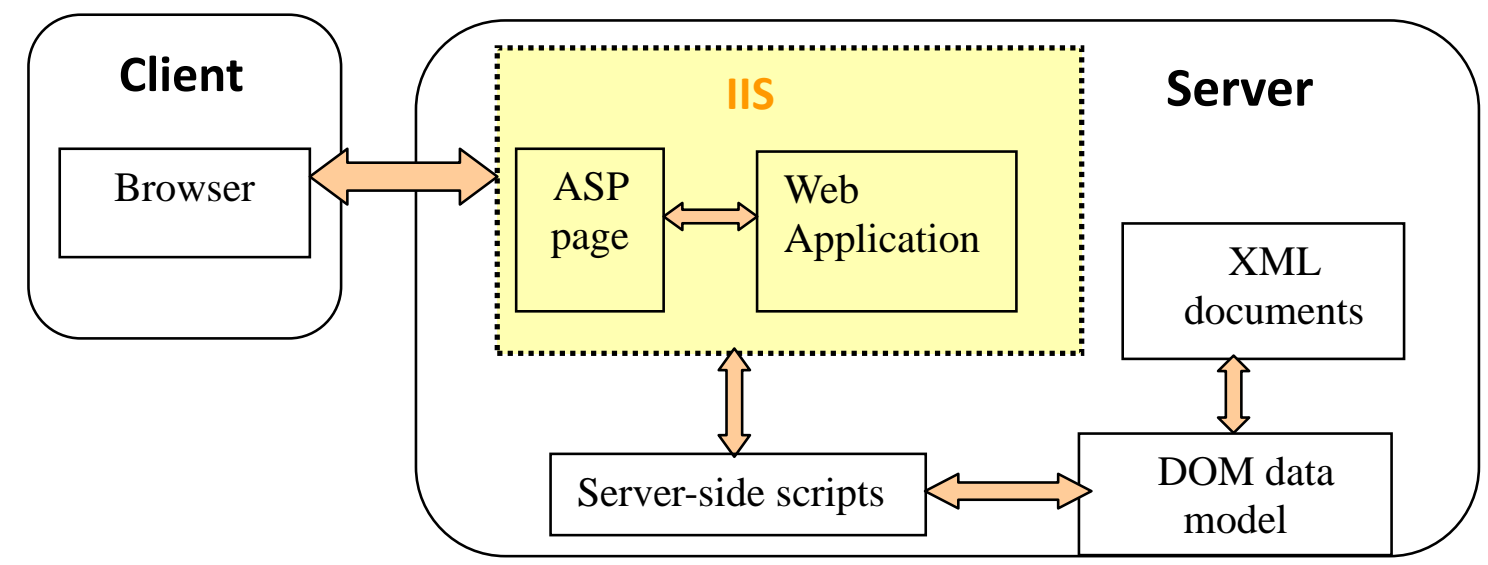

Figure 1. System architecture diagram

Function Module Design. System functions include user information management, view messages, edit messages, reply messages, delete messages Function module, the five modules is as shown below.

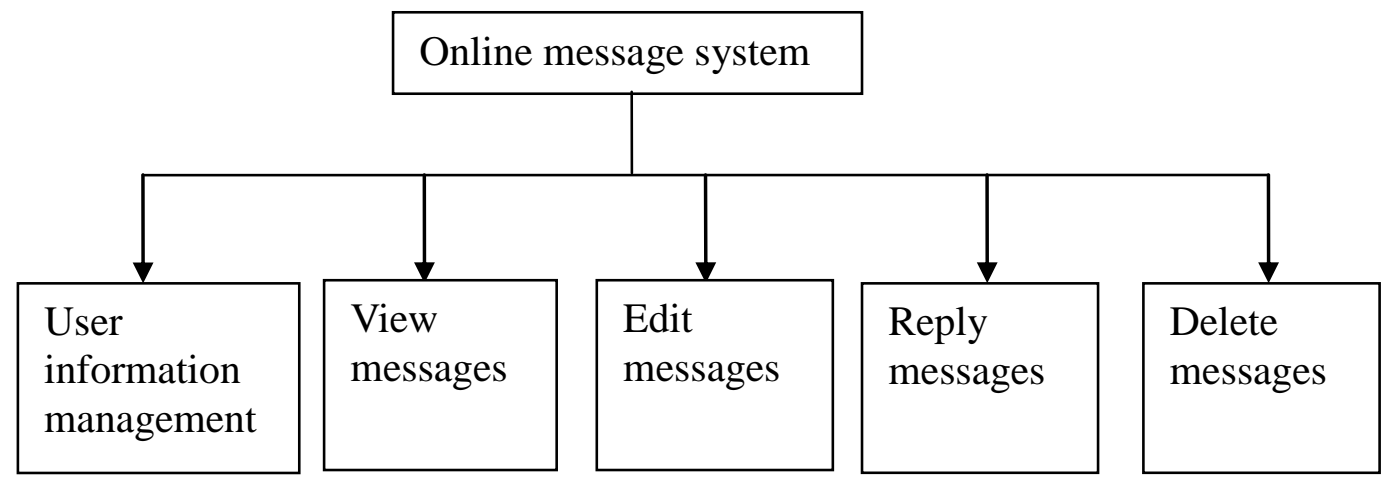

Figure 2. System functional module

\section{Key Technology}

Using XML to Store and Manage User Information and Message Information. The user information and message issued by each user are unified in the way of the child node. Message and its reply message are set as a child node. The corresponding XML document is as shown below:

$<$ ? $\mathrm{xml}$ version $=" 1.0$ " encoding="gb2312" ?>

$<$ XMLGuestBook >

$<$ item>

$<\mathrm{id}>001</ \mathrm{id}>$

$<$ username $>$ Zhangling $<$ /username $>$

$<$ gender $>$ male $<$ gender $>$

$<$ content $>$ Employment information $</$ content $>$

$<$ posttime $>2016-1-16</$ posttime $>$

$</$ item $>$

$<$ reply $>$ Thank you $</$ reply $>$

$</$ XMLGuestBook $>$

Way to View the User and Message. XML DOM Defines the standard method of accessing and handling XML documents [8], and the XML DOM document as a tree node tree (node-tree), each component of the document is a node [9]. For system development using XML document as the data source, DOM is an object oriented XML data interface [10]. Because the system uses ASP to implement web application, so ASP built-in object Server is needed to create an instance of the DOM object, and then import the XML document. The system created the Message.xml as data 
source document, the following code is to load the XML document:

$<\%$

strSourceFile = Server.MapPath("Message.xml") 'Get XML file path

Set objXML = Server.CreateObject("Microsoft.FreeThreadedXMLDOM")

'Create an object that operates XML

objXML.load(strSourceFile) ${ }^{`}$ Read XML file

$\%>$

Then read the required node information, which includes the membership of personal information and message information:

$<\%$

'StartNodes is start node

Set objRootsite $=$ objXML.documentElement.selectSingleNode

("XMLGuestBook")

id = objRootsite.childNodes.item(StartNodes).childNodes.item(0).text

username $=$ objRootsite . childNodes.item(StartNodes). childNodes.item(1).text

gender $=$ objRootsite.childNodes.item(StartNodes).childNodes.item(2).text

$\%>$

Edit and Reply Message. Through the above code, required node information stored in the XML file is read out, and then displayed on the page through the web application. When publishing message and saving the data to the XML file, the appendChild DOM method is needed to create the corresponding sub node item in the root node, the code is shown as follows:

$<\%$

Set

LNode=objXML.documentElement.selectSingleNode("XMLGuestBook

").AppendChild(objXML.createElement("item"))

Set DNode = LNode.appendChild(objXML.createElement("id"))

DNode. Text $=$ id

Set DNode = LNode.appendChild(objXML.createElement("username"))

DNode. Text $=$ username

objXML.save(strSourceFile)

$\%>$

Delete Message. All messages are stored in the XMLDOM document tree node, and each message corresponds to a child node, when achieving deleting module of online message system, firstly, it's needed to import XML source files, then using the nodes of the Id number to find the object to delete node belongs to the father item node, if the node is not empty, then DOM removeChild method is used to execute the delete operation.

Design Points. The system uses the popular online editing language UBB code to achieve the message editing and reply function. UBB code is referred as security code to replace HTML code in forum, the code uses regular expressions for matching, using the UBB editor can easily transform font, insert pictures, audio and video, insert rich emoticons, make the edit message more vivid, better show the characteristics of Web data.

\section{Conclusions}

The online message system based on XML DOM+ASP + UBB architecture is belonged to the B/S application system. The solution is convenient and effective in managing the network message, and has the characteristics of low cost, easy function expansion, convenient integration with existing systems, cross platform, easy maintenance, etc. The use of UBB code to achieve text editing features, you can insert pictures, animation, mathematical formula, can satisfy the diverse characteristics of Web information, at the same time the system is no longer based on HTML information display, but the true sense of information description and transfer, based on the Web for information sharing and retrieval has practical significance and practical value. 


\section{References}

[1] J. Feng, Q. Qian and Y.G Liao: Survey of Research on Native XML Databases, Application Research of Computers, Vol.15 (2006) No.6, p.1.

[2] S.C. Haw, C.S. Lee and M. Norwati: Bridging XML and Relational Databases: Mapping Choices and Performance Evaluation, IETE Technical Review, Vol.27 (2010) No.4, p.308.

[3] I. Lopez-Arevalo, V. Sosa-Sosa and F. Rojas-Lopez: Improving selection of synsets from WordNet for domain-specific word sense disambiguation, Computer Speech \& Language, Vol.6 (2016) No.3, p.132.

[4] Y.N. Li, Y.P. Wang and P. Jiang: Multi-objective optimization integration of query interfaces for the Deep Web based on attribute constraints, Data \& Knowledge Engineering, Vol.86 (2013), p.38.

[5] J.F. Zhou, Z.F. Bao and W. Wang: Efficient query processing for XML keyword queries based on the IDList index, The VLDB Journal, Vol.23 (2014) No.1, p.25.

[6] Q.S. Li, Q.Y. Wang and S. Wang: Query Understanding for XML Keyword Search, Journal of Software, Vol.23 (2012) No.8, p.2002.

[7] J.F. Zhou, X.F. Meng: Keyword Search on XML Data: A survey, Chinese Journal of Computers, Vol.35 (2012) No.12, and p.2459.

[8] L.J. Liu, C. Zhou and R.Wang: Suggestion of promising result types for XML keyword search. Proc.of the 13th Int''1 Conf.on Extending Database Technology. 2010

[9] T. Zheng, F. Duan: Discovering XML conditional functional dependencies based on content-aware, Application Research of Computers, Vol.31 (2014) No.12, p.3655.

[10]W. Li, X.F. Wang and J.F. Guo: Mining Spatial Frequently Changing Structures of XML, Chinese Journal of Computers, Vol.36 (2013) No.2, p.317. 